\title{
The Intramembrane Structure of Septate Junctions Based on Direct Freezing
}

\section{Citation}

Kachar, Bechara, Nicholas A. Christakis, Thomas S. Reese, and N. J. Lane. "The Intramembrane Structure of Septate Junctions Based on Direct Freezing." Journal of cell science 80, no. 1 (1986): 13-28.

\section{Permanent link}

http://nrs.harvard.edu/urn-3:HUL.InstRepos:33839962

\section{Terms of Use}

This article was downloaded from Harvard University's DASH repository, and is made available under the terms and conditions applicable to Other Posted Material, as set forth at http:// nrs.harvard.edu/urn-3:HUL.InstRepos:dash.current.terms-of-use\#LAA

\section{Share Your Story}

The Harvard community has made this article openly available.

Please share how this access benefits you. Submit a story.

Accessibility 


\title{
THE INTRAMEMBRANE STRUCTURE OF SEPTATE JUNCTIONS BASED ON DIRECT FREEZING
}

\author{
BECHARA KACHAR, NICHOLAS A. CHRISTAKIS, \\ THOMAS S. REESE
}

Laboratory of Neurobiology, NINCDS, NIH, Bethesda, Md 20205, U.S.A. AND NANCY J. LANE

A.F.R.C. Unit of Insect Neurophysiology and Pharmacology, Department of Zoology, University of Cambridge, Downing Street, Cambridge CB2 3E7, U.K.

\begin{abstract}
S U M M A RY
Smooth septate junctions from the midgut of the cricket, Acheta, and the horseshoe crab, Limulus, as well as Hydra-type septate junctions from the epidermis of $H y d r a$ have been studied by freeze-fracture after direct freezing using the liquid helium-cooled copper block/slam freezing method. The exoplasmic fracture face at both types of septate junction exhibits rows of closely packed but irregularly shaped intramembrane particles. Complementary to these particle rows, on the protoplasmic fracture face, are sharply defined grooves with a periodic variation in depth and width that was conspicuous in Hydra but less well defined in arthropods. The closely packed, irregular particles on the exoplasmic faces could represent plastically deformed portions of transmembrane proteins pulled through the bilayer during freeze-fracture. On the basis of this interpretation, the grooves on the protoplasmic faces represent a confluence of the bilayer disruptions occurring during fracturing. The structures observed here are different from those reported in replicas of glutaraldehyde-fixed and glycerol-cryoprotected tissue, in which the intramembrane junctional components partition with the protoplasmic face and often assume the appearance of continuous cylinders. This comparison illustrates some of the artifacts associated with freezefracturing and shadowing. On the basis of a comparison of freeze-fracture replicas and sections of lanthanum-infiltrated tissues, the relationship between intramembrane junctional components and intercellular septal elements is analysed.
\end{abstract}

\section{INTRODUCTION}

Membrane elements associated with intercellular junctions can be especially susceptible to changes induced by preparative procedures used in conventional freezefracture (Staehelin, 1974; van Deurs \& Luft, 1979; Pinto da Silva \& Kachar, 1982; Kachar \& Reese, 1982). Rapid freezing permits the investigation of the structural details of intercellular junctions without preliminary fixation or cryoprotection, providing preservation of the native structure, which is free of such artifacts. Furthermore, rapid freezing may facilitate alternative paths of fracture through junctions, revealing additional aspects of their structure. Such treatment of tight junctions in vertebrates has led to a reassessment of their nature (Kachar \& Reese, 1981, 1982; van Deurs, Bulow \& Mollgard, 1982); it appears that their structure may depend on lipids arranged as inverted cylindrical micelles and perhaps stabilized by peripheral protein moieties (Kachar \& Reese, 1982; Pinto da Silva \& Kachar, 1982).

Key words: septate junctions, intramembrane structure, direct freezing. 
Tight junction strands of various invertebrates, notably arthropods, also appear to be lipid-based, for the same reasons (Lane \& Swales, 1982; Swales \& Lane, 1983; Lane, 1984).

Smooth septate junctions found in the midgut and hepatopancreas of arthropods and many other invertebrates, as well as in insect Malpighian tubules (Flower \& Filshie, 1975; Dallai, 1976; Skaer, Harrison \& Lee, 1979; Lane \& Skaer, 1980; Noirot-Timothee \& Noirot, 1980), were at one time confused with vertebrate tight junctions (Noirot \& Noirot-Timothee, 1967), to which they were considered to be physiologically equivalent (Satir \& Gilula, 1973; Noirot-Timothee \& Noirot, 1980). This confusion resulted both from the structural similarities between the two in freeze-fracture replicas and from their putative functional similarity and evolutionary relationship (Green, 1978; Green, Bergquist \& Bullivant, 1979). Septate junctions, however, have been analysed little to date by rapid freezing methods, although a few studies have been made on unfixed, glycerinated specimens (Flower \& Filshie, 1975; Lane \& Harrison, 1978; Skaer et al. 1979; Lane \& Swales, 1982; Swales \& Lane, 1983). The pleated variety has been examined in insect central nervous system after rapid freezing by cryojet (Lane \& Swales, 1982; Swales \& Lane, 1983; Lane, 1984), while mention has been made of both pleated and smooth varieties in a recent report on insect Malpighian tubules rapidly frozen by plunging manually into liquid propane (Severs \& Green, 1983). The echinoderm double-septum septate junction has also been studied after direct freezing in supercooled nitrogen slush (Green, 1981, 1982).

The present report concerns the appearance of the smooth type of septate junction, as well as a similar type of septate junction found in Hydra (Wood, 1959, 1977; Filshie \& Flower, 1977; Wood \& Kuda, 1980), after fast freezing with no prior chemical treatment, using the highly effective liquid helium/slam freezing method (Heuser et al. 1979). Differences in fracture face distribution of the intramembrane components in conventionally prepared and fast-frozen tissue provides further opportunity for understanding the complex structure of these junctions; in particular, the relationship between intramembrane junctional components and intercellular septal elements as revealed by comparison of the freeze-fracture replicas with sections of lanthanum-infiltrated tissues. The high regularity and consistency of intramembrane components also facilitates identification of remaining artifacts associated with freeze-cleaving and replication. This paper gives a full presentation of results presented previously in abstract form (Kachar, Christakis, Reese \& Lane, 1983).

\section{MATERIALS AND METHODS}

Tissues from several invertebrates were examined, including the midgut from an insect, the cricket Acheta domestica, and from a primitive chelicerate arthropod, the horseshoe crab Limulus polyphemus. Hydra littoralis, was also studied in order to compare its septate junctions (Hand \& Gobel, 1972; Filshie \& Flower, 1977; Green, 1978; Wood \& Kuda, 1980) with the smooth septate junctions of arthropod midgut. The crickets were obtained from Wasco Lab., horseshoe crabs from the Marine Biological Laboratory, Woods Hole, MA, and Hydra from Carolina Biological Supply Co., Burlington, NC. 
Slices of midgut, quickly removed from each arthropod specimen, or intact Hydra were fast frozen on a liquid helium-cooled copper block (Heuser et al. 1979) with a time interval of about 5-10 s between removal of sample and freezing. Replicas were prepared by freeze-fracturing the specimen at $-110^{\circ} \mathrm{C}$ and at a vacuum of $10^{-f}$ Torr, shadowing with platinum and carbon, and backing the replica with carbon. The replicas were cleaned with sodium hypochlorite, mounted on grids, and examined in a JEOL $200 \mathrm{CX}$ or a Philips EM 420 . The micrographs are presented in the form of negative prints in which platinum deposits appear white.

Specimens were also fixed in buffered glutaraldehyde to which colloidal lanthanum had been added. These preparations were fixed for $1-16 \mathrm{~h}$ at room temperature in $2.5 \%$ glutaraldehyde in $0.1 \mathrm{M}$-cacodylate buffer, $\mathrm{pH} 7.2$, plus $6 \%$ sucrose and $2.5 \%$ lanthanum hydroxide (Revel \& Karnovsky, 1967). After fixation, the tissues were washed in buffer plus sucrose, then treated with buffered $1 \%$ osmium tetroxide for $1 \mathrm{~h}$. The material was then stained with aqueous uranyl acetate for $30 \mathrm{~min}$, dehydrated in an ascending series of ethanols to propylene oxide, and embedded in Araldite. Ultrathin sections were examined in a Philips EM 420.

\section{RESULTS}

\section{Limulus}

The Limulus midgut epithelial cells are joined by smooth septate junctions that appear in replicas as parallel rows of intramembrane strands separated from one another by $15-30 \mathrm{~nm}$ (Fig. 1). Individual strands appear on the exoplasmic (E) fracture face as rows of irregularly shaped intramembrane particles, while on the protoplasmic (P) fracture face they appear as continuous grooves (Figs 1, 2). A periodic variation in depth and width of these grooves is resolved in favourably shadowed areas (arrowheads, Fig. 2). The strands change orientation at tricellular junctions (Noirot-Timothé \& Noirot, 1980; Fristrom, 1982), where three cells meet (arrows, Fig. 2). The partition of intramembrane components of the individual strands of a tricellular septate junction is identical to that of intramembrane components at the regular strands (arrows, Fig. 2).

Intercellular septa belonging to individual septate junction strands are revealed as ribbon-like structures running between the plasma membranes in the junctional region. These septa are regularly aligned and separated by spaces $15-30 \mathrm{~nm}$ wide, which fill with lanthanum. The lanthanum in these spaces outlines circular structures (inset, Fig. 1), which are interpreted as columns extending between the cells. This appearance is characteristic of smooth septate junctions in Limulus (see Lane \& Harrison, 1978) and in invertebrates in general (Lane \& Skaer, 1980). No intramembrane representation of these columns was apparent in freeze-fracture replicas.

\section{Acheta}

Septate junctions in the cricket (Acheta) midgut epithelial cells generally appear as strands of various lengths meandering over the membrane fracture face (Fig. 3 ). Two or more strands are frequently closely associated side by side. Individual strands appear as sequences of irregular particles, 6-10 nm in diameter, on the E face, and as continuous grooves on the $P$ face (Fig. 3). A similar partition of intramembrane components also occurs at the tricellular junctions in the cricket (Figs 4-6). The tricellular junctions in the cricket display, in addition, short filaments cross-bridging the two most lateral septate junction strands at the corner of 
each cell. These filaments lie on the $\mathrm{E}$ face (arrowheads, Fig. 5); they correspond to grooves on the $\mathrm{P}$ face that connect the continuous, most lateral strands of the tricellular junction (Fig. 4). A complex and irregular partition of the tricellular junctional elements is obtained when the fracture plane jumps from the interior of the membrane of one cell to the interior of the membrane of a neighbouring cell, cross-fracturing the junction septum at one of the strands (arrows, Fig. 6).

Lanthanum impregnation of this portion of the junctional strands is accessible from the extracellular spaces. The septate junctions in cricket midgut are identified by the distribution of septal ribbons (Fig. 7). In many examples, the septal ribbons lie next to each other. The junctions in the cricket did not display interseptal columns, but septal ribbons had a subtle periodic substructure (arrowheads, Fig. 7). This substructure of the septal strands within the intercellular cleft was also suggested by views depending on fortuitous planes of freeze-fracture where the fracture jumps from the membrane of one cell to the membrane of an adjacent cell exactly at the septate junction strand (Fig. 8). The periodicity of this substructure (3-4 nm) does not correlate with the periodicity of intramembrane particles on the exoplasmic fracture face, which have an $8-10 \mathrm{~nm}$ periodicity.

\section{Hydra}

The observations in Hydra were limited to epidermal cells because adequate freezing was achieved only at the surface of the specimen. Septate junctions, formed by parallel arrays of strands, join the lateral plasma membranes of these epithelial cells, forming a band several micrometres wide close to the exterior surface of each cell (Wood, 1959, 1977). The partition of individual strands on the $\mathrm{P}$ and $\mathrm{E}$ faces (Figs 9, 10) is similar to that at smooth septate junctions from the insect midgut (particles on the $\mathrm{E}$ face; continuous grooves on the $\mathrm{P}$ face). However, the grooves on the $P$ face (Fig. 10) exhibit a periodic (8-9 $\mathrm{nm}$ centre-to-centre) variation in depth and width that is more striking than that which occurs in the arthropods. This periodicity coincides with the periodicity of the particles cleaved with the E face (Fig. 9).

Lanthanum-infiltrated preparations of the Hydra septate junctions reveal parallel arrays of septal ribbons with no intervening columns (inset, Fig. 9). The lines of contact of abutting apical membranes from adjacent cells, at the surface of the

Fig. 1. Smooth septate junctions from the midgut of Limulus represented by rows of irregular intramembrane particles (IMPs) on the exoplasmic $(E)$ fracture face, and corresponding grooves in the protoplasmic $(P)$ fracture face. Some IMPs lie in the P-face grooves. Inset: lanthanum-infiltrated smooth septate junction from Limulus midgut revealing undulating rows of septal ribbons $(s)$ with intercalated stain-filled extracellular spaces. Clear round areas in the lanthanum-filled space are interpreted as silhouettes of intercellular columns (see the text) that have no clear representation in membrane fracture faces. $\times 220000$; inset, $\times 210000$.

Fig. 2. Fracture revealing $P$ faces of two cells at a tricellular region of a smooth septate junction from Limulus midgut, where three cells meet (arrows). Here the intramembrane components of the junction appear as continuous P-face grooves identical to those in the rest of the junction. In favourably shadowed areas the grooves exhibit a periodic variation in depth and width (arrowheads). $\times 200000$. 

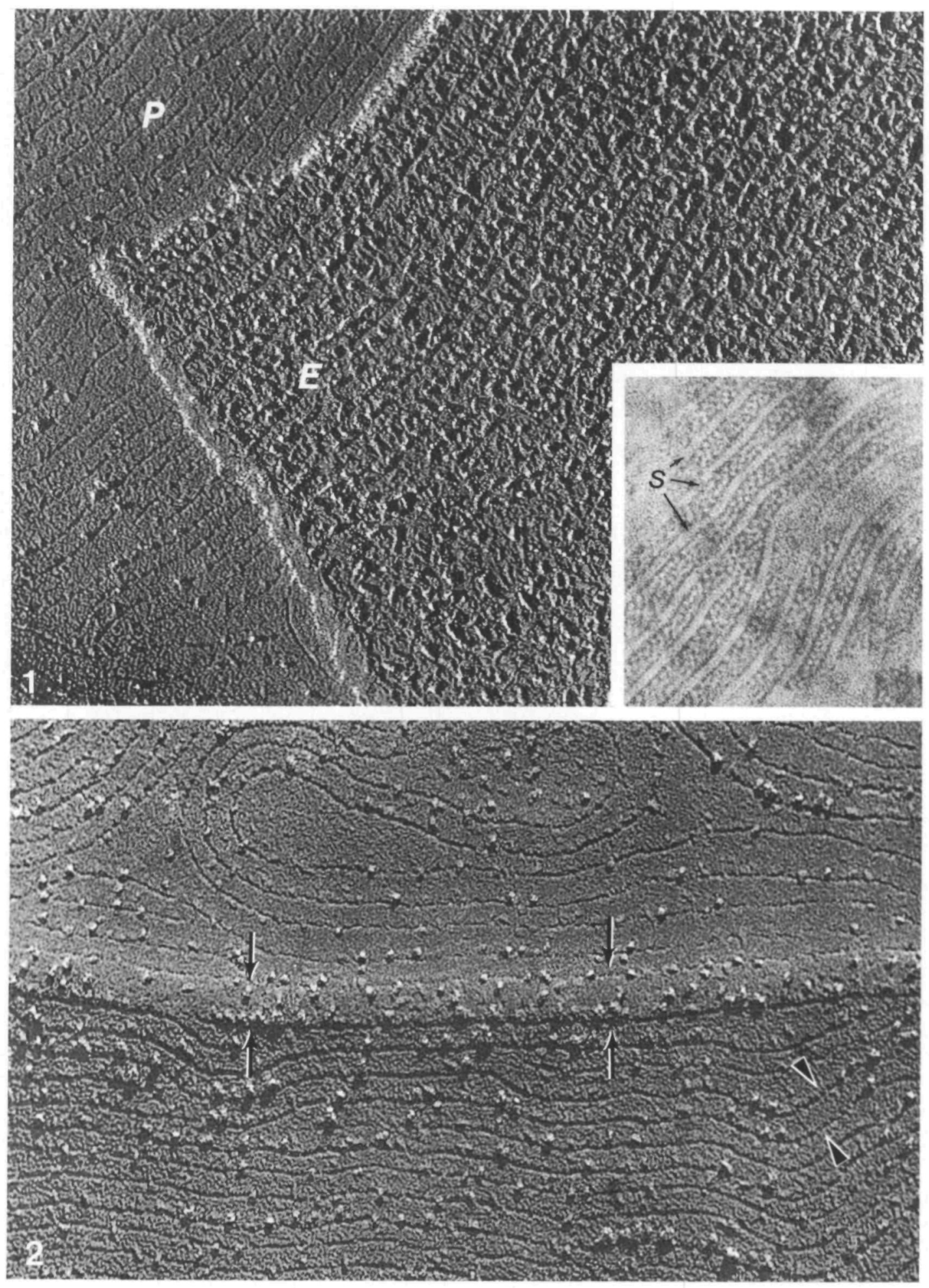


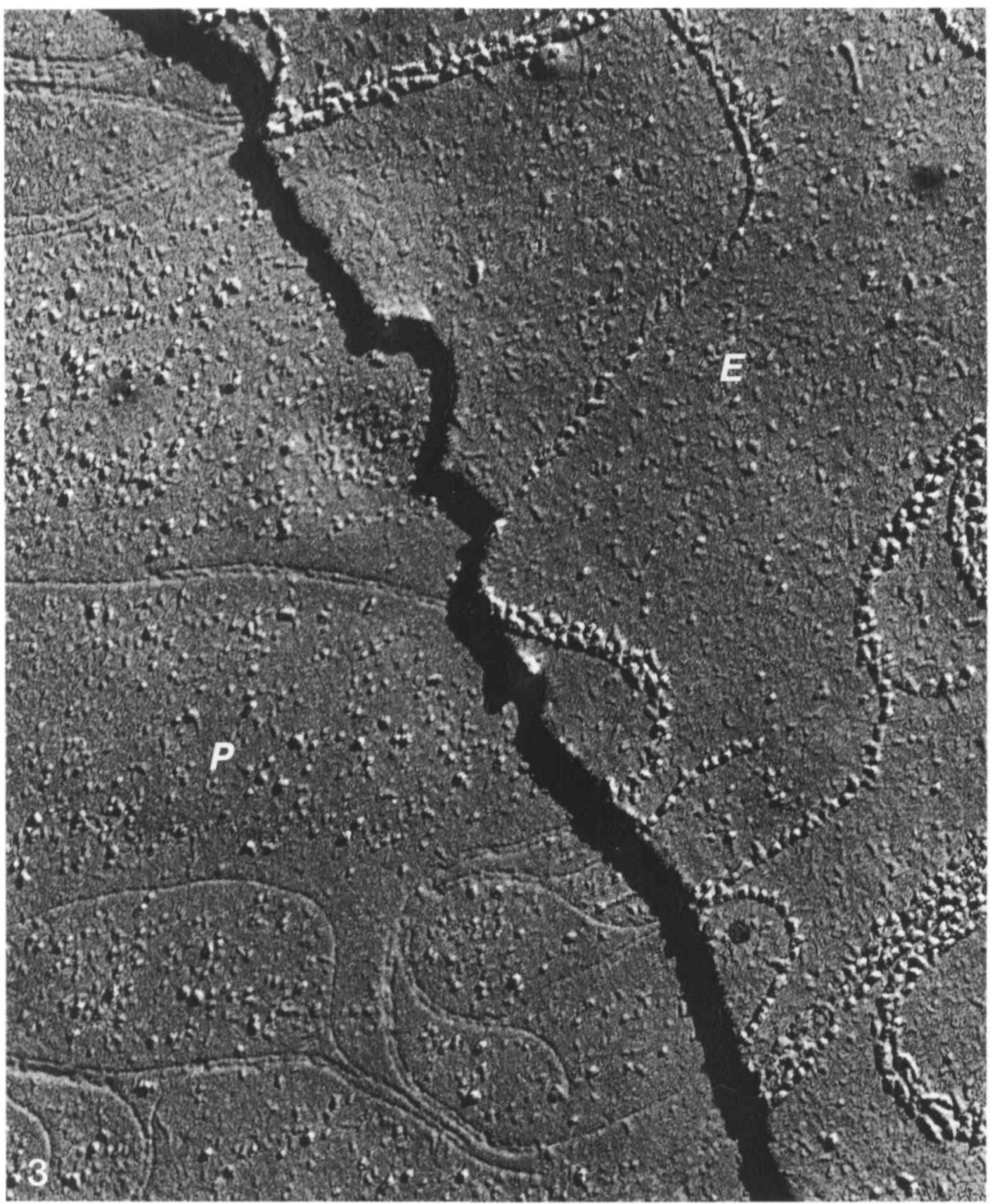

Fig. 3. Smooth septate junctions from the cricket midgut; strands appear on the E face $(E)$ as meandering rows of IMPs, which are sometimes in close association with other IMP rows. Septate junction strands on the $\mathrm{P}$ face $(P)$ appear as corresponding continuous grooves; at fracture face transitions the grooves are coincident with the IMP rows on $\mathrm{E}$ faces. $\times 140000$. 

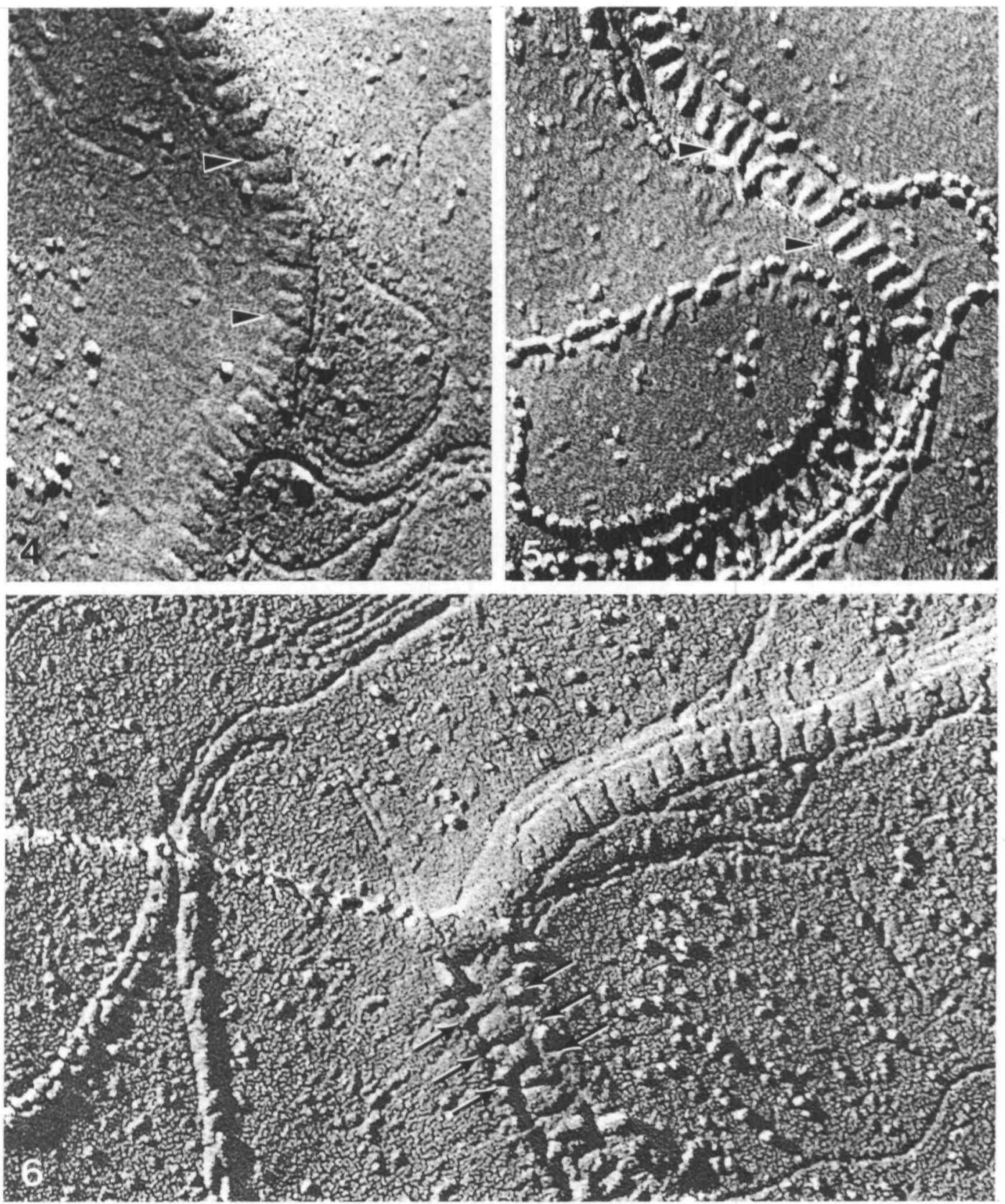

Figs 4, 5, 6. Tricellular junctions from cricket midgut. Individual components appear as short grooves in the protoplasmic face (Fig. 4) and particle rows on the E face (Fig. 5). In addition, E-fracture faces display short filaments cross-bridging the two most lateral septate junction strands at the corner of each cell (arrowheads, Fig. 5). During fracture these cross-bridging filaments leave a complementary groove on the $\mathrm{P}$ face (Fig. 4, arrowheads). Fig. 6 shows the complex partition of components that occurs when the plane of fracture jumps from a $\mathrm{P}$ face to an $\mathrm{E}$ face at a tricellular junction (arrows). Fig. 4, $\times 230000$; Fig. $5, \times 230000$; Fig. $6, \times 230000$. 

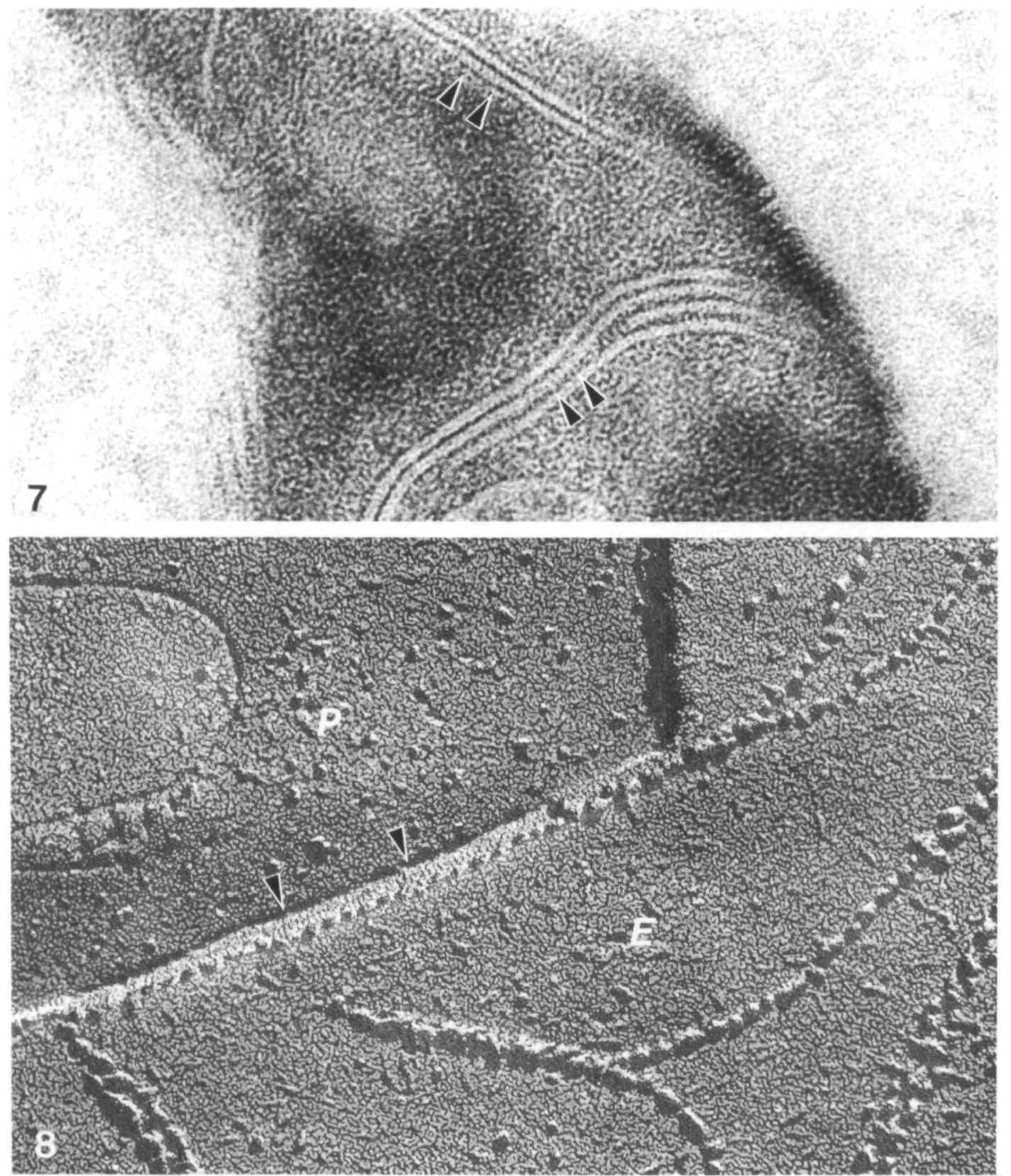

Fig. 7. Lanthanum-impregnated cricket midgut showing the irregular arrangement of the septal ribbons in the intercellular space, which is just the arrangement predicted from the freeze-fracture views (see Figs 3-6). The intercellular components of the strand frequently show a fine periodicity (arrowheads), suggestive of the regular substructure seen in replicas (see Fig. 8). Interseptal columns are not evident. $\times 240000$.

Fig. 8. Cricket midgut septate junction showing a fortuitous cleavage plane where the fracture jumped from the $\mathrm{P}$ face $(P)$ of one membrane to the $\mathrm{E}$ face $(E)$ of the adjacent membrane at a septate junctional strand. This view uncovers the septum itself (arrowheads); the alignment of the septum with the intramembrane particle strand shows they are closely associated. $\times 220000$. 
Hydra, are occasionally disrupted, allowing the intercellular cleft to splay open and expose the outermost septa strands (Figs 11, 12). The P fracture faces of these 'uncoupled' strands (positively identified by the presence of ice overlaying the junction; e.g. see Fig. 11) is similar to the regular intact strands at the lateral plasma membranes, where two cells are in contact. Moreover, what may be assumed to be the septa in Hydra (Figs 12,13) show a regularity in packing, which can be better correlated with the array of intramembrane particle rows seen at the $\mathrm{E}$ fracture face than is possible in the midgut junctions. The periodic cleft structures bridging the intercellular cleft are seen more readily when the junction is not splayed open so the most external septal strand, nearest to the outer surface of the animal, is exposed by freeze-fracture (arrowheads, Fig. 13).

\section{General features}

In all three tissues, the intramembrane components of septate junction strands appear as chains of irregular particles that cleave with the $\mathrm{E}$ fracture face. However, in some regions of the replicas, where the angle between the direction of shadow and the septate junction strand was perpendicular, individual particles are not resolved. Instead, the intramembrane components of the junction appear as segments of an irregular cylinder. This effect of the direction of shadow upon the appearance of the junction is illustrated in Figs 14, 15. Particles in the same region of the junction become resolved when the shadow direction is parallel to the strand, but appear fused into a continuous bar where the strand changes direction to become perpendicular to the direction of shadow.

\section{DISCUSSION}

We have examined tissues from three different invertebrates, which have been prepared by fast freezing. The different types of septate junction strands have in common a ribbon-like septum, which spans the intercellular space and contacts aligned rows of intramembrane elements in the adjacent plasma membranes. The nature of the intramembrane elements, the extent to which their presence modifies the continuous bilayer structure and the relationship of these elements to the intercellular septa must be considered.

\section{Structure of the intramembrane elements}

The smooth septate junctions in the cricket and horseshoe crab as well as the $H y d r a$-type junctions in Hydra are characterized by rows of closely packed, irregularly shaped intramembrane particles, which position on the exoplasmic (E) fracture face after direct freezing. The complementary protoplasmic (P) face displays complementary grooves with a periodic variation in depth and width, which is conspicuous in Hydra but less well defined in arthropods. Comparable fracturing of junctional particles onto the $\mathrm{E}$ face has been reported in unfixed, glycerinated preparations of smooth septate junctions (Flower \& Filshie, 1975; Lane \& Harrison, 1978; Skaer et al. 1979), while echinoderm septate junctions, frozen directly in 

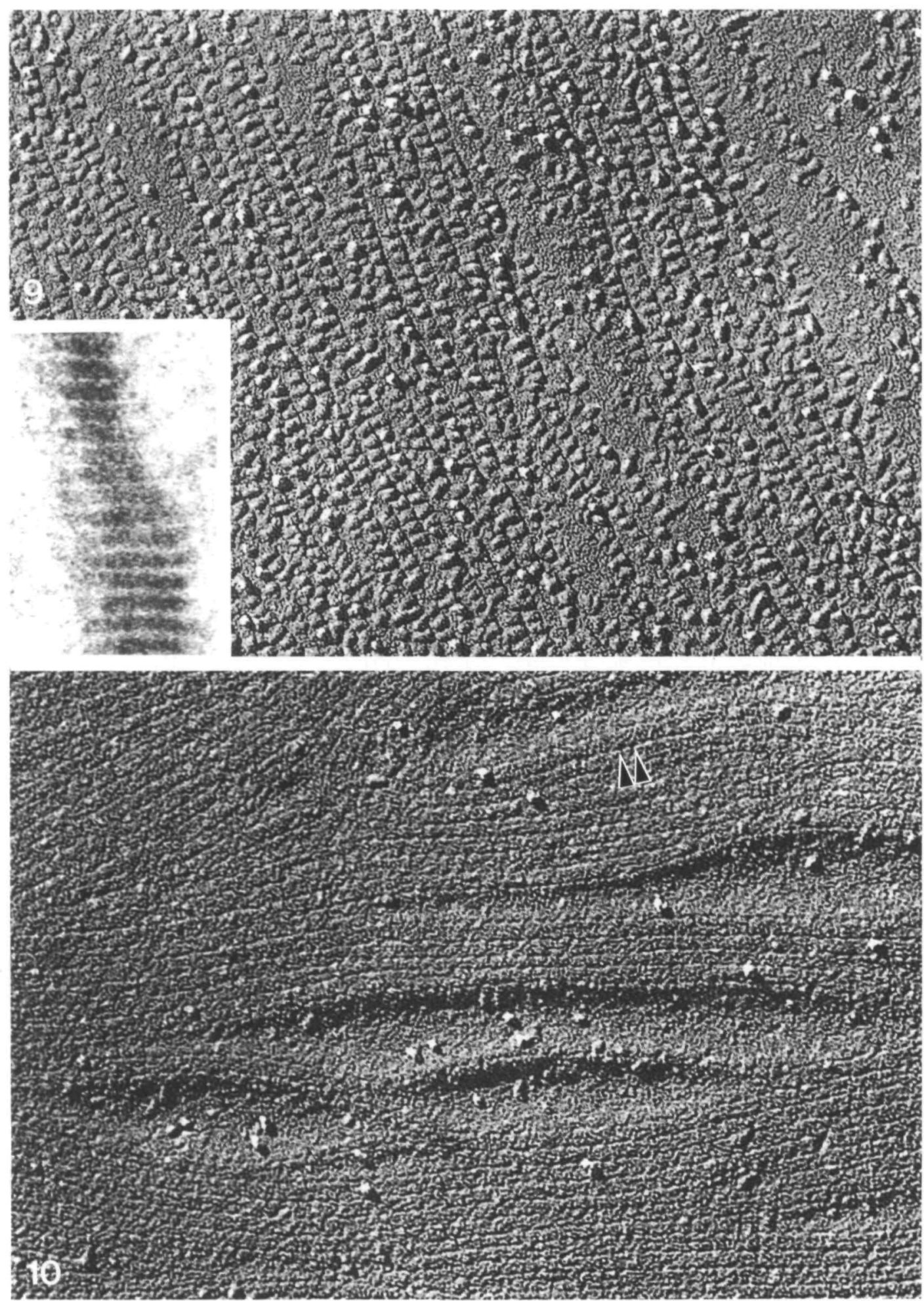
subcooled liquid nitrogen, also exibit P-face grooves with periodic pits (Green, 1981, 1982). The replicas of these unfixed, echinoderm septate junctions fast-frozen in nitrogen slush display particles that also partition with the $\mathrm{E}$ face and are aligned in rows; these, however, appear to be double or are to be found as short rods (Green, 1981,1982 ), in contrast to the separate particles observed in this study. Interestingly, these echinoderm structures occasionally display particles appearing as small bars lying at right angles to the row in which they occur, as also have been found here, particularly in Hydra epidermis.

Fixation and, or, cryoprotection may result in intramembrane particle translocation that appears not to be due to plastic deformation (Green, 1981, 1982); this may result in separation of these structures from the intermembrane septal components. The fact that septate junction strands occasionally appear to be continuous cylinders is ascribed to a confluence of the shadow covering adjacent particles; this appearance was common in some replicas where the angle of shadow was perpendicular to the row of particles, but the complete reason for this effect is not clear. It may be that individual particles contain a number of fine branches, 'tree-like' in shape, that represent plastically deformed transmembrane proteins pulled out of the membrane (Pinto da Silva et al. 1981). Plastic deformation would explain the irregular shape of these membrane particles. A chain of closely packed, irregular intramembrane particles is, according to this explanation, the actual configuration of the fastfrozen septate junction elements that appear on the $\mathrm{E}$ fracture face.

The grooves on the protoplasmic fracture face are not precisely complementary to the chains of particles on the exoplasmic fracture face. Thus, structural alterations must also occur, during or after fracture, in one or both of the fracture faces. The continuous grooves could represent confluence of bilayer disruptions that occur when the row of transmembrane proteins is pulled out during fracture. Furthermore, the tops of intramembrane particles would be seen in the $\mathrm{P}$ faces, whereas structures at the membrane midplane, where the intramembrane particles in rows would be closest to each other, would be seen on the $\mathrm{E}$ face. Thus, the periodic variation in depth and width of the grooves, which is most conspicuous in Hydra, may be a more accurate representation of the shapes of individual transmembrane proteins at the level of the membrane midplane. The complementarity of this periodicity to the junctional particles seen on the $\mathrm{E}$ face supports this interpretation.

The structures observed here are different from those in replicas of fixed septate junctions, in which the junctional components tend to remain on the $P$ face (Flower \& Filshie, 1975) to which they may be cross-linked (Van Deurs \& Luft, 1979). Presumably the particles in the closely packed rows, which often appear to be fused laterally into ridges in the fixed smooth septate junctions, are also each cross-linked to

Figs 9, 10. Septate junctions from the epidermis of Hydra showing rows of IMPs on the E-fracture face (Fig. 9) and grooves on the $P$ face (Fig. 10). The grooves have a periodic punctate variation in depth and width (arrowheads, Fig. 10). Inset: lanthanuminfiltrated septate junction between epidermal cells of $H y d r a$, showing septal strands in an en face view; interseptal columns are lacking. Fig. 9, $\times 200000$; Fig. 10, $\times 200000$; inset, $\times 210000$. 

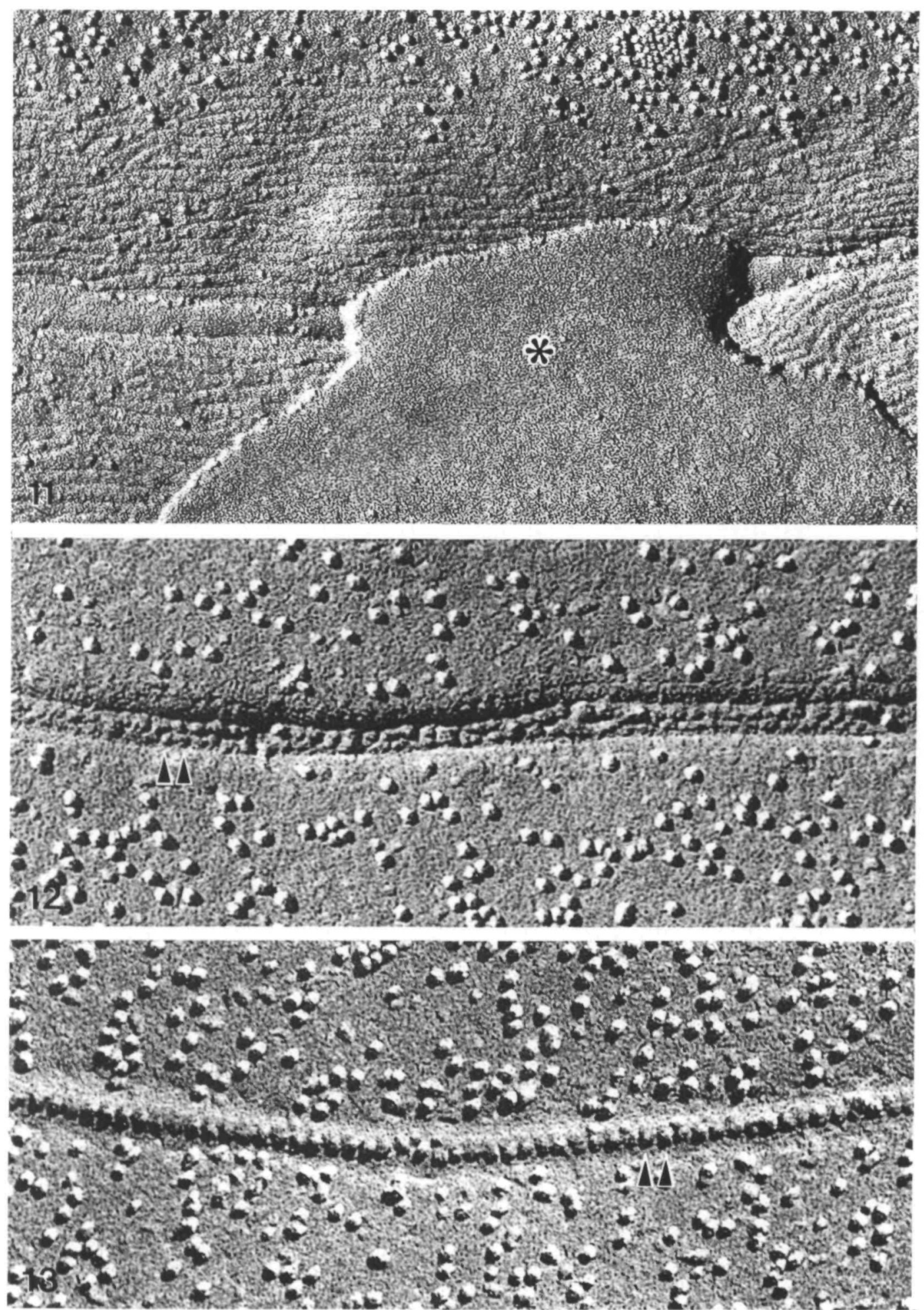
one another by glutaraldehyde fixation to produce the observed smooth intramembrane strands. The appearance of the junctional strands in rapidly frozen preparations, which is not that of continuous strands on the $\mathrm{P}$ face, but is instead of irregular particles on the $E$ face, suggests that the native structure of septate junctions is based on transmembrane particulate structures, rather than major modifications of the bilayer structure itself.

The detailed morphology of fast-frozen septate junctions differs from that of fastfrozen vertebrate tight junctions, which appear (in the absence of cross-linking fixatives) as continuous intramembrane cylinders, intercalated within the hydrophobic domain of the bilayer and partitioning with either the P or E face (Kachar \&
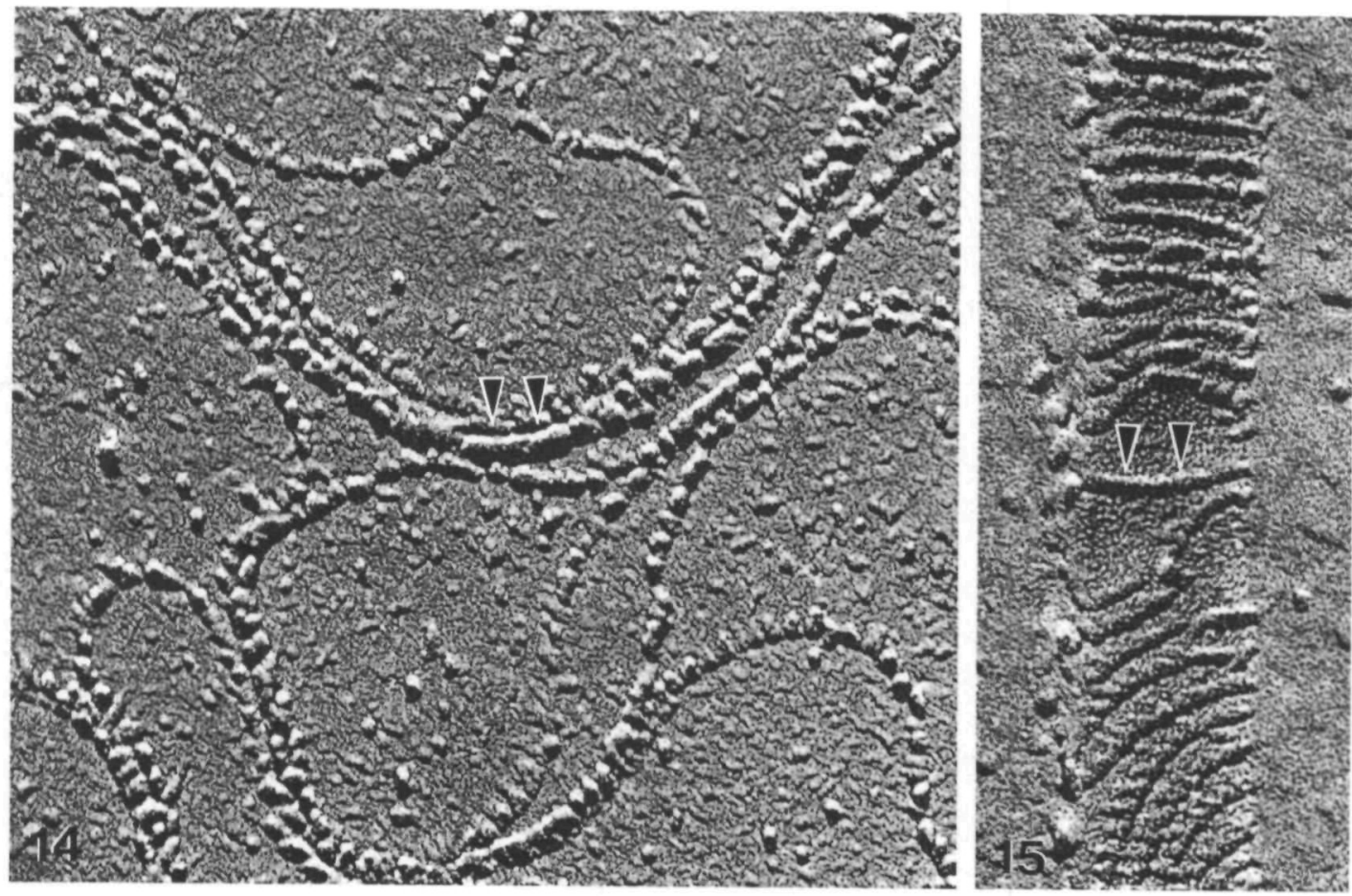

Figs 14, 15. Cricket midgut (Fig. 14) and Hydra epidermis (Fig. 15) showing the effect of the direction of shadow on the appearance of the intramembrane components of the septate junctions. IMPs that appear discrete when the shadow angle is parallel to the strand, appear confluent (at arrowheads) when the shadow angle is perpendicular to the strand. Fig. 14, ×170000; Fig. 15, ×170000.

Figs 11, 12, 13. Abutting membranes at the apices of adjacent epidermal cells in Hydra may splay out, presumably with disruption of the outermost septa. The presence of ice overlying the junction (asterisk) in Fig. 11 identifies these as membranes of cells facing a fluid space. The P-face grooves retain their appearance (Figs 11, 12), even though they can no longer be sites of attachment to an adjacent cell. This plane of fracture also reveals periodic structures cross-bridging the clefts (Fig. 12), which must be part of the septum. The periodic nature of the septal structures cross-bridging the intercellular cleft is readily appreciated when the outermost septal strand is at the apex of the intercellular apposition (arrowheads, Fig. 13). Fig. 11, $\times 150000$; Fig. 12, $\times 230000 ;$ Fig. 13, $\times 230000$. 
Reese, 1982). The tight-junction strands are accompanied by complementary grooves that show no periodic variation in width and depth even after freezing at slow rates, when the intramembrane cylinders appear beaded (Kachar \& Reese, 1982). Fast-frozen intramembrane cylinders at tight junctions have a smooth surface and a diameter similar to that of inverted cylindrical micelles of lipids (Kachar \& Reese, 1982). There is a potential interruption of the lipidic continuum of the exoplasmic half of the plasma membrane across the tight junction (Dragsten, Blumental \& Handler, 1981), but the freeze-fracture images provide evidence of fusion of the exoplasmic halves of neighbouring cells (Pinto da Silva \& Kachar, 1982; Kachar \& Reese, 1982, 1983). Thus, the configuration of the lipid bilayer appears to be radically altered at tight-junction strands, but there is no indication that this occurs at septate-junction strands.

\section{Relationship of septal structure to intramembrane elements}

After lanthanum impregnation, the intercellular septa of individual septatejunction strands are revealed as ribbon-like structures bridging the plasma membranes at the junction. In Limulus the septa are regularly aligned and in the spaces between them are the columns typical of smooth septate junctions (Lane \& Skaer, 1980 ). We do not see in the freeze-fracture replicas any clear intramembrane representation of these columns. They are, therefore, likely to be extrinsic to the membrane. Close packing of the parallel aligned junctional ridges, as seen in both cricket midgut and Hydra epidermis, is permitted by the absence of columns between the septa. The intramembrane particle rows and intercellular septa seem to have exactly the same distribution and structure in all the species, and hence they may represent different aspects of the same structure. The particle ridges, perhaps, are intramembrane anchoring sites for the septa.

Subunits of the septal ribbon are visualized in Limulus as well as in Hydra and Acheta. In Hydra they show a regularity in packing that can be correlated with the intramembrane particle rows seen at the exoplasmic fracture face. Such periodic structures cross-bridging the intercellular cleft are more readily seen at the most external septal strand, nearest to the outer surface of the animal. When the septa in Hydra are disrupted, the intramembrane structure in each plasma membrane seems to remain intact so that the intramembrane backbone of the junctions may not depend on septal integrity. The septal ribbon itself could form by the pairing of projecting surface components of integral membrane proteins represented by the rows of intramembrane particles on the $E$ face. In cricket, however, the septal ribbons appear to have a substructure that does not correlate with the periodicity of intramembrane particles on the $\mathrm{E}$ face. This lack of direct correspondence between the intramembrane elements and the subunits of the septal ribbon still does not exclude the possibility that the septal elements are extensions of the integral membrane proteins seen in the freeze-fracture replicas.

The discontinuous nature of the septal ribbons, as indicated by the substructures visualized in replicas as well as in lanthanum-impregnated tissues, correlates with recent physiological evidence that individual septate junctions are leaky, even to 
molecules as large as inulin (O'Donnell, Maddrell \& Gardiner, 1983). Furthermore, characterization of the modifications in the regular structure of the septate junctions at the site where three cells meet (tricellular junctions) remains elusive, so the tricellular points could provide another source of leakage. In the cricket, however, very conspicuous short filaments cross-bridge the two most lateral septate junction strands at the corner of each cell. The height of these bridges seen on the $E$ face is much greater than the thickness of the bilayer leaflet, so these bridges must protrude into the interior of the cell. The continuous groove that they leave on the complementary fracture face may represent a disruption of the protoplasmic leaflet of the bilayer during fracture. The inner surface components of septate junction strands and their possible relationship to cytoskeletal components is currently being investigated in rapid-frozen, freeze-etched specimens.

We are grateful to Dr John Walrond for assistance with some of the rapid freezing, and to $\mathrm{Mr}$ J. B. Harrison and $\mathrm{Mr}$ W. M. Lee for assistance with sectioning and photography.

\section{REFERENCES}

DAllai, R. (1976). Septate and continuous junctions associated in the same epithelium. 7. submicrosc. Cytol. 8, 163-174.

Dragsten, P. R., Blumental, R. \& Handler, J. S. (1981). Membrane asymmetry in epithelia: Is the tight junction a barrier to diffusion in the plasma membrane? Nature, Lond. U294, $718-722$.

Filshie, B. K. \& Flower, N. E. (1977). Junctional structures in Hydra. F. Cell Sci. 23, $151-172$.

FLower, N. E. \& FilshiE, B. K. (1975). Junctional structures in the midgut cells of lepidopteran caterpillars. F. Cell Sci. 17, 221-239.

Fristrom, D. K. (1982). Septate junctions in imaginal disks of Drosophila: a model for the redistribution of septa during cell rearrangement. $\mathcal{7}$. Cell Biol. 94, 77-87.

GreEN, C. R. (1978). Variations of septate junction structure in the invertebrates. 9th Int. Conf. Electron Microsc. Toronto, vol. II, Biology (ed. J. M. Sturgess), pp. 338-339. Canada: The Imperial Press.

Green, C. R. (1981). Fixation-induced intramembrane particle movement demonstrated in freeze-fracture replicas of a new type of septate junction in echinoderm epithelia. $\mathcal{F}$. Ultrastruct. Res. 75, 11-22.

GreEN, C. R. (1982). Intramembrane particle movement revealed by study of an intercellular junction. 7. Microsc. 125, 201-206.

Green, C. R., Bergquist, P. R. \& Bullivant, S. (1979). An anastomosing septate junction in endothelial cells of the phylum Echinodermata. 7. Ultrastruct. Res. 67, 72-80.

HAND, A. R. \& GoBEL, S. (1972). The structural organization of the septate and gap junctions of. Hydra. F. Cell Biol. 52, 397-408.

Heuser, J. E., Reese, T. S., Dennis, M. J., Jan, Y., JaN, L. \& Evans, L. (1979). Synaptic vesicle exocytosis captured by quick freezing and correlated with quantal transmitter release. F. Cell Biol. 81, 275-300.

Kachar, B., Christakis, N., Reese, T. S. \& Lane, N. J. (1983). An interpretation of the intramembrane structure of septate junctions based on direct freezing. $\mathcal{~}$. Cell Biol. 97, 80A, 306.

KaCHAR, B. \& ReESE, T. S. (1981). Membrane structure at tight junctions in quick frozen tissue. J. Cell Biol. 91, 123A.

KaCHAR, B. \& REESE, T. S. (1982). Evidence for the lipid nature of tight junction strands. Nature, Lond. 296, 464-466.

KACHAR, B. \& REESE, T. S. (1983). Formation of misplaced and reflexive tight junction strands in prostate epithelial cells. F. Ultrastruct. Res. 82, 90-95.

LANE, N. J. (1984). A comparison of the construction of intercellular junctions in the CNS of vertebrates and invertebrates. Trends Neurosci. 7, 95-99. 
LANE, N. J. \& HarRison, J. B. (1978). An unusual type of continuous junction in Limulus. 7. Ultrastruct. Res. 64, 85-97.

LANE, N. J. \& SKaER, H. LE B. (1980). Intercellular junctions in insect tissues. Adv. Insect Physiol. $15,35-213$.

LANE, N. J. \& SwaLes, L. S. (1982). Liquid propane-rapid jet-freezing of intercellular junctions in insect CNS. F. Cell Biol. 95, 92A.

NoIrot, C. \& NoIRot-TimothEe, C. (1967). Un nouveau type de jontion intercellulaire (zonula continua) dans l'intestin moyen des Insectes. C. r. hebd. Séanc. Acad. Sci., Paris, ser. D, 264, 2796-2798.

NoIrot-Timothé, C. \& NoIRot, C. (1980). Septate and scalariform junctions in arthropods. Int. Rev. Cytol. 63, 97-140.

O'Donnell, M. J., Maddrell, S. H. P. \& Gardiner, B. O. C. (1984). Passage of solutes through the walls of the Malpighian tubules of Rhodnius by paracellular and transcellular routes. Am. 7. Physiol. 246, R759-R769.

Pinto da Silva, P. \& Kachar, B. (1982). On tight-junction structure. Cell 28, 441-450.

Pinto da Silva, P., Kachar, B., Torrisi, M. R., Brown, C. \& Parkinson, C. (1981). Freezefracture cytochemistry: Replicas of critical point-dried cells and tissues after fracture-label. Science, N.Y. 213, 230-233.

REVEL, J.-P. \& KARNOVSKY, M. J. (1967). Hexagonal array of subunits in intercellular junctions of the mouse heart and liver. F. Cell Biol. 33, $\mathrm{C} 7-\mathrm{C} 12$.

SATIR, P. \& Gilula, N. B. (1973). The fine structure of membranes and intercellular communication in insects. A. Rev. Ent. 18, 143-166.

SEvers, N. J. \& GreEN, C. R. (1983). Rapid freezing of unpretreated tissues for freeze-fracture electron microscopy. Biol. Cell 47, 193-204.

Skaer, H. LE B., Harrison, J. B. \& LeE, W. M. (1979). Topographical variations in the structure of the smooth septate junction. F. Cell Sci. 37, 373-389.

Staehelin, L. A. (1974). Structure and function of intercellular junctions. Int. Rev. Cytol 39, 191-283.

SWALES, L. S. \& LANE, N. J. (1983). Insect intercellular junctions: rapid-freezing by jet propane. 7. Cell Sci. 62, 223-236.

van Deurs, B. \& LuFT, J. H. (1979). Effects of glutaraldehyde fixation on the structure of tight junctions. A quantitative freeze-fracture analysis. F. Ultrastruct. Res. 68, 160-172.

van Deurs, B., von Bulow, F. \& Mollgard, K. (1982). Tight junctions: Architecture of plasma membranes as sites of cell-cell "fusion" in relation to paracellular permeability. Tokai exp. clin. Med. 7 (Suppl), 197-202.

WoOD, R. L. (1959). Intercellular attachment in the epithelium of Hydra as revealed by electron microscopy. F. biophys. biochem. Cytol. 6, 343-352.

WOOD, R. L. (1977). The cell junctions of Hydra as viewed by freeze-fracture replication. 7. Ultrastnuct. Res. 58, 299-315.

Wood, R. L. \& KUDA, A. M. (1980). Formation of junctions in regenerating Hydra: septate junctions. J. Ultrastruct. Res. 70, 104-117.

(Received 3 April 1985 -Accepted, in revised form, 17 fuly 1985) 